\title{
УПРАВЛЕНИЕ ВИРТУАЛЬНОЙ ИНФРАСТРУКТУРОЙ ОБЛАЧНЫХ РЕШЕНИЙ ІААS
}

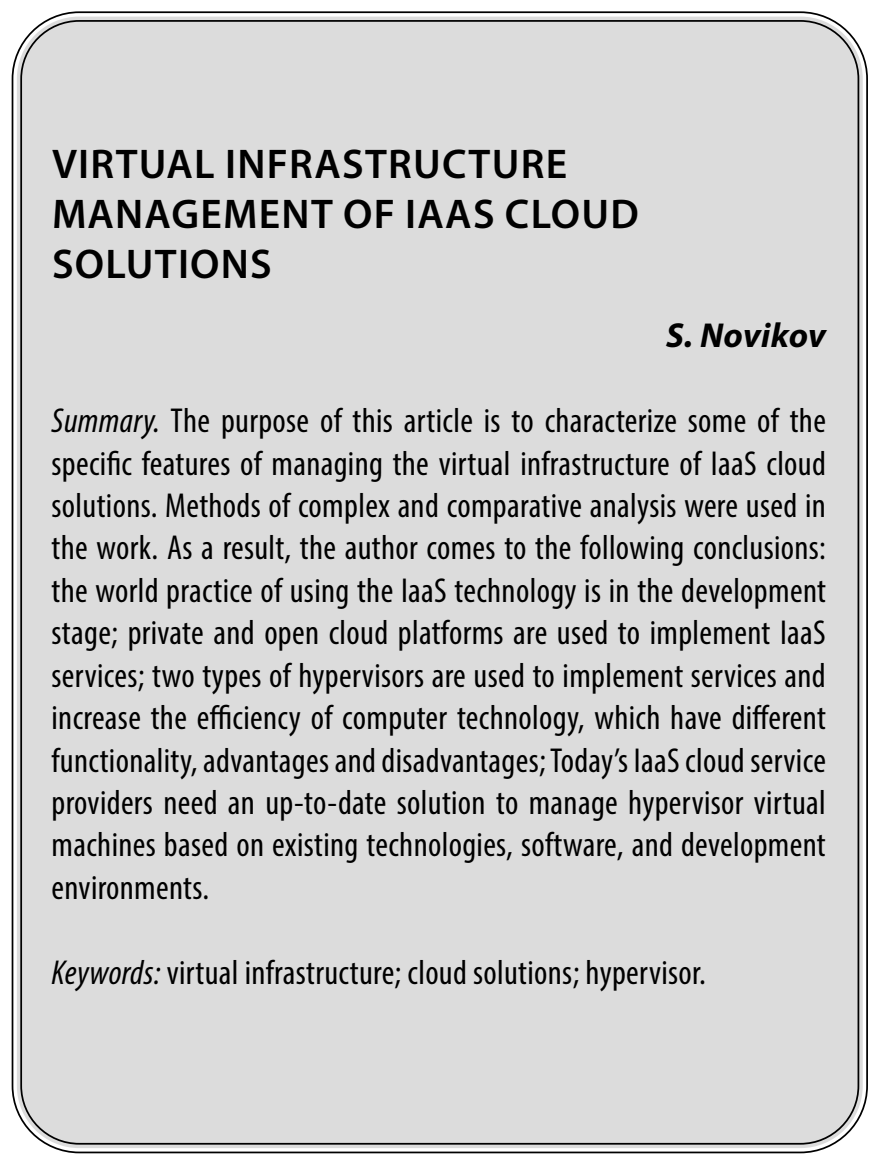

C овременный глобальный рынок облачных технологий интенсивно растет. Для него характерны следующие тенденции: быстрый рост расходов на публичные и частные сервисы, а также сопутствующего рынка услуг, ЦОДов и трафика данных в таких системах [5]. Согласно прогнозам исследовательской консалтинговой компании «Gartner», рынок облачных сервисов будет увеличиваться на 17,5\% ежегодно, в 2022 году превысит объем индустрии ИТ-услуг, а к 2025 году достигнет 832,1 млрд. долл. По оценкам «IDC», развитие российского рынка опередит общемировые темпы за счет работы отечественных поставщиков облачных решений на основе контейнерной виртуализации [2]. В 2025 году его объем составит 2,51 млрд. долл. [6].

Среди крупнейших сегментов рынка особое место занимают инфраструктурные сервисы laaS (англ. Infrastructure-as-a-Service). Инфраструктура как услуга представляет собой модель обслуживания в облачных
Новиков Сергей Владимирович

Уральский федеральный университет,

2. Екатеринбург

novserg@mail.ru

Аннотация. Цель данной статьи - охарактеризовать некоторые специфические особенности управления виртуальной инфраструктурой облачных решений laaS. B работе использованы методы комплексного и сравнительного анализа. В результате автор приходит к выводам: мировая практика использования технологии laaS находится в стадии развития; для внедрения сервисов ІаaS используются закрытые и открытые облачные платформы; для реализации сервисов и увеличения эффективности использования вычислительной техники используются гипервизоры двух типов, которые имеют разные функциональные возможности, достоинства и недостатки; современным компаниям-провайдерам облачных laaS-услуг необходимо актуальное решение, позволяющее управлять виртуальными машинами гипервизора с учетом существующих технологий, программных средств и сред разработки.

Ключевые слова: виртуальная инфраструктура; облачные решения; гипервизор.

вычислениях, предоставляющую потребителям по подписке фундаментальные информационно-технологические ресурсы - виртуальные серверы с заданной вычислительной мощностью, операционной системой (чаще всего предустановленной провайдером из шаблона) и доступом к сети. В мировой практике облачных вычислений накопился положительный опыт использования технологии laaS, которая продолжает интенсивно развиваться. Ее востребованность связана с заинтересованностью отраслевых компаний в использовании платформенных облачных решений для повышения эффективности своей деятельности, а также с формированием соответствующих партнерских экосистем, что будет способствовать масштабированию бизнеса провайдеров услуг [6]. По прогнозам аналитиков «Gartner», к 2022 году стоимость laaS-сегмента достигнет 80,98 млрд. долл. [8].

Управление виртуальной инфраструктурой входит в число базовых задач при использовании любых об- 
Таблица 1. Облачные платформы управления виртуальными дата-центрами, основанные на закрытых и открытых решениях

\begin{tabular}{|c|c|c|c|}
\hline \multicolumn{2}{|l|}{ Закрытые решения } & \multicolumn{2}{|l|}{ Открытые решения } \\
\hline $\begin{array}{l}\text { Amazon Web Services } \\
\text { (AWS) }\end{array}$ & $\begin{array}{l}\text { Коммерческое публичное облако, } \\
\text { поддерживаемое и развиваемое } \\
\text { компанией Amazon }\end{array}$ & Apache CloudStack & $\begin{array}{l}\text { Универсальная платформа управления } \\
\text { средами выполнения виртуальных машин }\end{array}$ \\
\hline $\begin{array}{l}\text { Google Cloud Platform } \\
\text { (GCP) }\end{array}$ & $\begin{array}{l}\text { Набор облачных служб, которые } \\
\text { выполняются на инфраструктуре } \\
\text { Google }\end{array}$ & OpenStack & $\begin{array}{l}\text { Облачная платформа, помогающая } \\
\text { создавать программно-конфигурируемые } \\
\text { ЦОДы }\end{array}$ \\
\hline Microsoft Azure & $\begin{array}{l}\text { Облачная платформа компании } \\
\text { Microsoft для разработки, выполнения } \\
\text { приложений и хранения данных } \\
\text { на серверах, расположенных } \\
\text { в распределённых дата-центрах }\end{array}$ & OpenNebula & $\begin{array}{l}\text { Открытая платформа для организации } \\
\text { управления cloud-инфраструктурой } \\
\text { и виртуальными окружениями }\end{array}$ \\
\hline
\end{tabular}

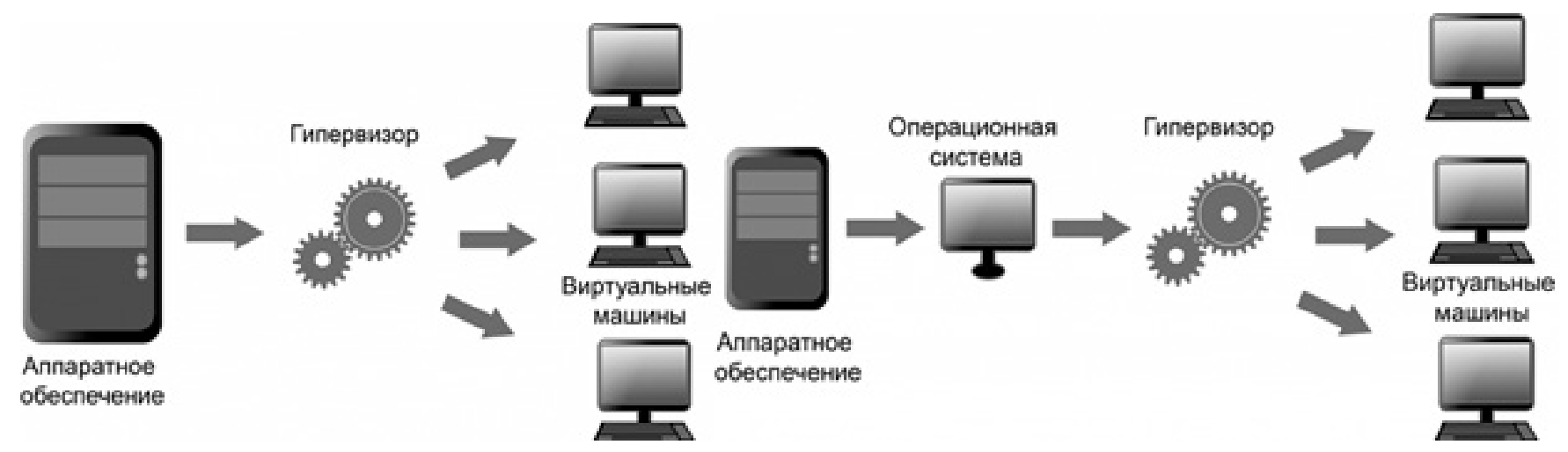

Рис. 1. Принцип работы гипервизоров 1-го и 2-го типов

лачных сервисов, поэтому для внедрения и реализации laaS-технологий используются разнообразные технические, системные и языковые платформы. Существующие решения условно можно разделить на две группы (таблица 1). Первую группу составляют проприетарные, закрытые решения крупных корпораций. К известным платформам управления виртуальными дата-центрами, основанным на закрытых решениях, относятся: Amazon Web Services (AWS), Google Cloud Platform (GCP), Microsoft Azure [8]. Вторую группу составляют открытые платформы с применением собственного серверного оборудования. Наиболее часто используемыми открытыми системами для построения виртуальных дата-центров считаются Apache CloudStack, OpenStack, OpenNebula [3]. Провайдеры накапливают объем предоставляемых сервисов, конкурируя по параметрам качества и набора услуг.
Развитие технологий виртуализации, а также широкое применение облачных вычислений и инфраструктурных сервисов laaS в работе отраслевых компаний способствовали появлению большого числа платформ серверной виртуализации - гипервизоров, представляющих собой программы или аппаратные схемы, обеспечивающие одновременное, параллельное выполнение нескольких операционных систем на одном и том же хост-компьютере, их изоляцию друг от друга, защиту, безопасность, разделение ресурсов между различными запущенными ОС и управление ресурсами [7;9]. Основным преимуществом гипервизоров является увеличение эффективности использования вычислительной техники, которая выражается в оптимальной утилизации вычислительных ресурсов за счет снижения времени простоя серверного оборудования и повышения плотности виртуальных машин. 
Таблица 2. Сравнительный анализ гипервизоров 1-го и 2-го типов

\begin{tabular}{|c|c|}
\hline Название платформы & Характеристика платформ серверной виртуализации \\
\hline Microsoft Hyper-V & $\begin{array}{l}\text { Microsoft Hyper-V - система виртуализации от корпорации Microsoft. Hyper-V } \\
\text { является гипервизором, работающим на микроядерной архитектуре. Прямой доступ } \\
\text { к оборудованию имеет только хостовая операционная система. Распределением ресурсов } \\
\text { и прочими задачами занимается гипервизор. }\end{array}$ \\
\hline KVM & $\begin{array}{l}\text { KVM (Kernel-based Virtual Machine) - система аппаратной виртуализации в среде Linux. } \\
\text { Является загружаемым модулем ядра Linux (kvm.ko). Состоит из модуля ядра kvm.ko } \\
\text { и процессорно-специфических модулей kvm-intel.ko и kvm-amd.ko. }\end{array}$ \\
\hline Citrix Hypervisor & $\begin{array}{l}\text { Citrix Hypervisor (ранее XenServer) - это коммерческая платформа серверной } \\
\text { виртуализации, имеющая несколько версий: Free, Standard и Enterprise. Отличительной } \\
\text { особенностью платформы является поддержка адаптации ядра гостевой операционной } \\
\text { системы для работы совместно с гипервизором (паравиртуализации) наряду с аппаратной } \\
\text { виртуализацией. }\end{array}$ \\
\hline Oracle VM VirtualBox & $\begin{array}{l}\text { VirtualBox - программный продукт виртуализации, разработанный компанией Oracle. } \\
\text { Работает под управлением всех популярных операционных систем, так как Windows, Linux, } \\
\text { MacOS. Присутствует возможность проброса USB на гостевую операционную систему. }\end{array}$ \\
\hline
\end{tabular}

Существует два типа платформ серверной виртуализации (рисунок 1) [4]. Гипервизоры первого типа запускаются непосредственно через аппаратное обеспечение и не требуют установки какой-либо операционной системы. Для работы монитора виртуальных машин второго типа нужна операционная система, так как через нее производится доступ к аппаратной части. Согласно мнению экспертов, лучшим платформами серверной виртуализации считаются гипервизоры первого типа, поскольку они работают напрямую с оборудованием и обеспечивают более высокую производительность.

Среди программных продуктов аппаратной виртуализации интерес представляют платформы Microsoft Hyper-V, VMware ESX, KVM (Kernel-based Virtual Machine), Citrix Hypervisor (ранее XenServer), VirtualBox (таблица 2).

При выборе платформ серверной виртуализации важно учитывать целый ряд разнообразных факторов. Основным средством для создания, удаления виртуальных машин, изменения их параметров и мониторинга являются инструменты, поставляемые в составе того или иного гипервизора. Однако в некоторых случаях применение штатных инструментов управления не представляется возможным [1]. Наибольшей состав- ляющей конечной совокупной стоимости владения того или иного технологического решения является стоимость лицензий на программное обеспечение, реализующее функции гипервизоров. Поэтому использование свободного ПО снизит капитальные затраты на внедрение виртуализации, что увеличит экономическую эффективность. При этом необходимо заметить, что программное обеспечение, не требующее оплаты за лицензии, значительно сложнее в развертывании и сопровождении, так как требует более высокой квалификации специалистов и менее развитую техническую поддержку.

Очевидно, что существующим компаниям-провайдерам облачных laaS-услуг, предоставляющим своим клиентам выделенные виртуальные сервера для использования по модели аренды, необходимо новое технологическое решение, которое должно позволять пользователю управлять своими виртуальными ресурсами и в то же время ограничивать его доступ к операционной системе самого гипервизора. В связи с этим актуальной становится разработка сервиса, работающего отдельно от среды управления гипервизора и позволяющего обеспечить унифицированный двухсторонний механизм взаимодействия с виртуальными пользовательскими ресурсами.

\section{ЛИТЕРАТУРА}

1. Бастани Кеннет, Лонг Джош. Java в облаке. Spring Boot, Spring Cloud, Cloud Foundry. — Питер, 2019. — 624 c.

2. Будущее облачных вычислений [Электронный ресурc].— URL: https://sbercloud.interfax.ru/posts/budushhee-oblachnyh-vychislenij (дата обращения: 11.10.2021)

3. Обзор платформ для построения облаков [Электронный ресурc].—URL: https://habr.com/ru/post/140375/ (дата обращения: 12.10.2021)

4. Пастухов Д.А., Юрчик П.Ф. Сравнительный анализ гипервизоров // Автоматизация и управление в технических системах. - 2014. — № 4.—C. 129140. 
5. Провайдеры ІааS ведут борьбу за европейский рынок — обсуждаем ситуацию и события индустрии [Электронный pecypc]. — URL: https://habr.com/ ru/company/1cloud/blog/502354/ (дата обращения: 11.10.2021)

6. Рынок облачных услуг России перевалил за миллиард долларов [Электронный ресурc].——URL: https://www.cnews.ru/news/top/2020-10-20_rynok_ oblachnyh_uslug_rossii (дата обращения: 12.10.2021)

7. Chris Wolf, Erick M. Halter. Virtualization: From the Desktop to the Enterprise. Apress, 2005.

8. Global Public Cloud Revenue to Grow to $\$ 258 \mathrm{~B}$ in 2020, SaaS to Surpass laaS by over \$100B [Электронный pecypc].— URL: https://stockapps.com/ blog/2020/11/04/global-public-cloud-revenue-to-grow-to-258b-in-2020-saas-to-surpass-iaas-by-over-100b/?wpmeteordisable=1 (дата обращения: 12.10.2021)

9. Rajkumar Buyya, Maria A. Rodriguez. Cost-Efficient Orchestration of Containers in Clouds: A Vision, Architectural Elements, and Future Directions [Электронный ресурc].—URL: https://arxiv.org/abs/1807.03578 (дата обращения: 12.10.2021)

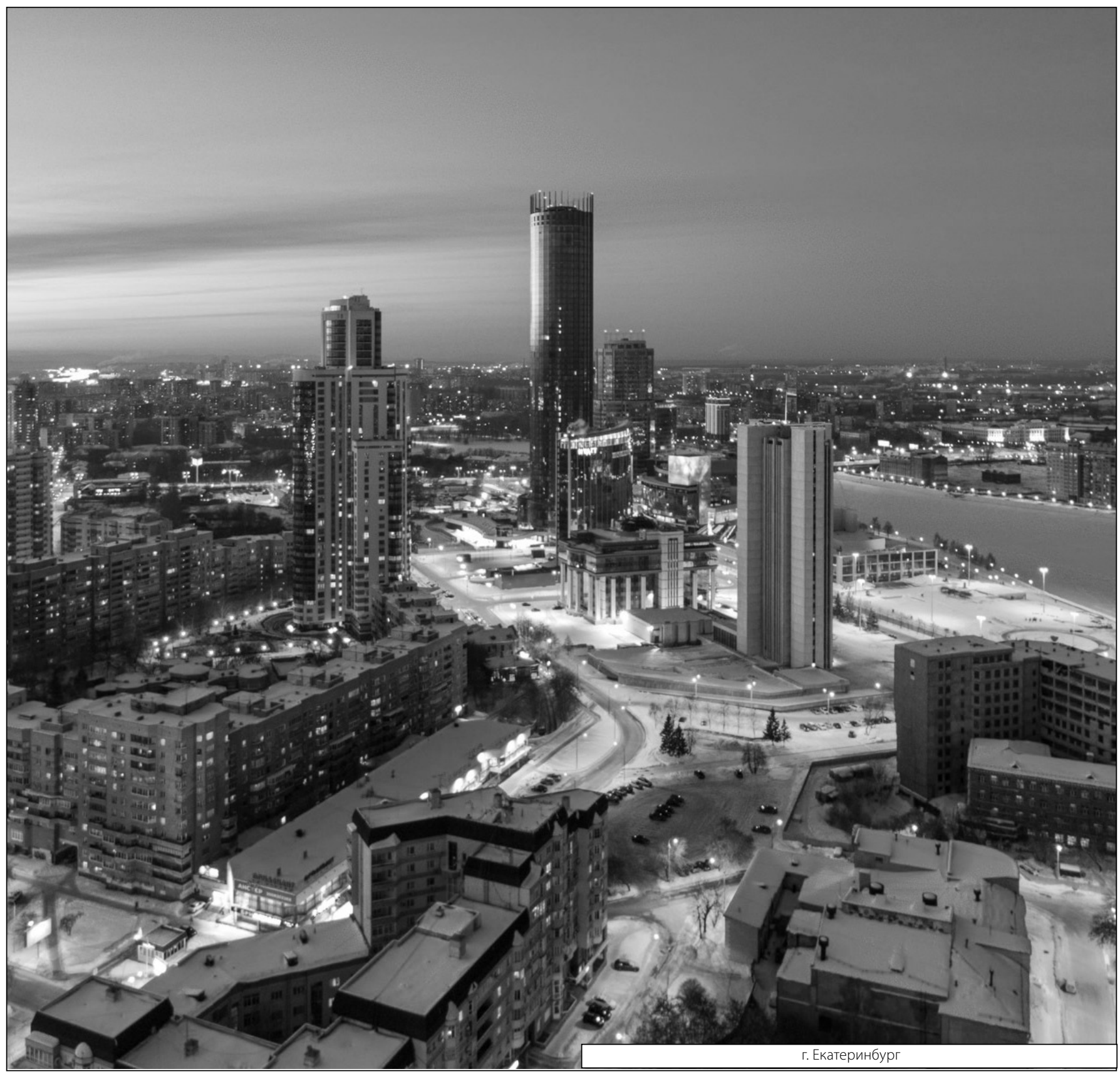

